\title{
Synchronized Phasors Monitoring During the Islanding Maneuver of an Active Distribution Network
}

\author{
Alberto Borghetti, Senior Member, IEEE, Carlo Alberto Nucci, Fellow, IEEE, Mario Paolone, Senior Member, IEEE, \\ Gaetano Ciappi, and Aurelio Solari
}

\begin{abstract}
The paper describes the performances of a phasor measurement unit (PMU) prototype based on a synchrophasor estimation algorithm conceived for the monitoring of active distribution networks, as well as its experimental application during some intentional islanding and reconnection tests of an urban medium voltage power network. With respect to typical applications in transmission networks, the use of PMUs in distribution networks requires very low values of total vector error (TVE), which involves particular low values of phase errors of the synchrophasor estimates. These requirements are met by a specifically developed PMU, the characteristics and experimental characterization of which are illustrated in the paper. Three of these PMUs have been then used to monitor experimental tests carried out to assess the capability of a urban distribution network to operate autonomously when fed by a local 80 MW combined-cycle power plant. The information provided by the installed PMUs significantly facilitate the operator maneuvers and appear to be useful for the development of an improved control and management system of the active distribution network.
\end{abstract}

Index Terms-Active distribution networks, islanding maneuver, phasor measurement unit (PMU), synchrophasor uncertainties.

\section{INTRODUCTION}

D ISTRIBUTED monitoring of synchronized phasors, nowadays essential for the operation of modern power transmission networks, may also represents a useful tool to improve the control flexibility of energy management systems of distribution networks. The concept of "active distribution networks" applies to those distribution networks in which embedded generation is actively used by the energy management system in order to achieve the operation objectives (i.e., [1], [2]). Bidirectional flows within distribution level and the upstream network, intentional islanding, autonomous islanded operation and back-synchronization, are operating conditions and maneuvers that characterize active distribution networks.

Manuscript received February 12, 2010; revised May 12, 2010, August 19, 2010; accepted October 25, 2010. Date of publication January 06, 2011; date of current version February 18, 2011. This work is supported in part by HERA S.p.A., Italy. A preliminary version of this paper was presented at the IEEE Innovative Smart Grid Technologies Conference, Gaithersburg, MD, Jan. 19-21, 2010. Paper no. TSG-00027-2010.

A. Borghetti, C. A. Nucci, and M. Paolone are with the University of Bologna, Italy (e-mail: alberto.borghetti@unibo.it; carloalberto.nucci@unibo.it; mario. paolone@unibo.it).

G. Ciappi and A. Solari are with HERA S.p.A., 59100 Prato, Italy (e-mail:gaetano.ciappi@gruppohera.it; aurelio.solari@gruppohera.it).

Digital Object Identifier 10.1109/TSG.2010.2094213
As shown in the literature, some functions to be implemented in the management system of active distribution networks appear somehow similar those already applied for the operation of transmission networks. In this respect, also the concept of a distributed monitoring system based on phasors measurement units (PMUs) can be one of these functions [3].

As known, PMUs provide the measurements of voltage/current phasors synchronized with high precision time references that, in general, is provided by the UTC-GPS one [11]-[13].

Significant work has been already carried out in order to apply PMUs in distribution networks. Typical application fields are: protection functions, such as loss of main (e.g., [4], [5]) and fault event monitoring [6], [7], state estimation (e.g., [8]), synchronous islanded operation [9] and for power quality monitoring (e.g., [10]).

However, the peculiar characteristics of distribution networks compared to transmission ones (such as reduced line lengths and limited power flows) result, in general, into very small phase differences between voltage phasors of different busses. Therefore, very low values of the uncertainty relevant to the phasor-phase estimation is required, hindering the direct utilization of PMUs developed for transmission networks applications [14]. An additional important constraint related to PMUs application into distribution networks is the need of specific synchrophasor estimation algorithms unaffected by the presence of high levels of harmonic distortion.

Typical algorithms implemented into PMUs are based on the direct estimation of the phasor amplitude, frequency and phase provided by the application of the discrete Fourier transform (DFT) to the sampled waveform. This approach has been also proposed in the literature for the development of PMUs to be applied in distribution networks. For example, the measurement procedure proposed in [15] is based on a sample reallocation procedure and on the DFT application by using both a fixed and adjustable-length time window. However, the standard deviation of the error distribution of the synchrophasor phase estimates obtained in [15] is in the order of $0.3 \mathrm{mrad}$. This uncertainty level appears excessive for the PMU application in distribution networks.

The synchrophasor estimation algorithm described and adopted in this paper, the preliminary version of which was presented in [14], is based on a combined use of DFT tone identification algorithm and a subsequent time-domain analysis aimed at improving the PMU accuracy. 
The developed DFT-based tone-reconstruction algorithm allows the achievement of high levels of PMU accuracy, namely a standard deviation of the synchrophasor phase error distribution in the order of $0.07 \mathrm{mrad}$, significantly lower than the values reported in the literature as obtained with other approaches. Moreover, the algorithm accuracy is robust with respect to typical harmonic distortion levels in distribution systems.

The main aim of the paper is to discuss the experimental application of the developed PMUs during islanding and back-synchronization maneuvers of an active distribution network in order to show the capabilities of these devices to support the system operators during these maneuvers. The monitored network is composed by an 80-MW power plant that supplies, through a cable subtransmission link, an urban distribution system.

The structure of the paper is the following. Section II describes: i) the general requirements of synchrophasor estimation in distribution systems; ii) the algorithm implemented in the developed PMU; and iii) the PMU experimental characterization. Section III illustrates the structure and the characteristics of the power system where the islanding tests have been carried out. Section IV describes the earlier mentioned maneuvers and presents the experimental results obtained by using the developed PMUs. Section V concludes the paper.

\section{PMU REQUIREMENTS AND CHARACTERISTICS}

In view of the different characteristics of distribution networks compared to transmission ones, the main peculiar requirements for PMUs to be used at the distribution level are (e.g., [15]):

i) lower values of total vector error (TVE), RMS, and, above all, phase uncertainties;

ii) robustness of the PMU performances in presence of distorted input signals.

Moreover, the application of PMUs to monitor slow electromechanical transients, characterized by nonnegligible deviations from the nominal frequency, could involve, as discussed in [13], [17], and [18], ${ }^{1}$ an important misestimation of the synchrophasor phases and frequencies and calls for the following three additional requirements [18]:

iii) use of consecutive independent observation windows in order to reach an accurate estimate of the electromechanical transient frequencies;

iv) estimation of the frequency using a sufficient number of phase angle measurements with a reasonable span of 3-6 cycles of the nominal frequency;

v) frequency estimation with the same time-tag used for the phasor estimation.

The proposed synchrophasor estimation procedure has been specifically conceived with the aim of satisfying all the above five requirements.

\footnotetext{
${ }^{1}$ In spite of that, IEEE Std. C37.118 [16] defines the performance requirements of PMUs in steady state conditions without providing detailed information for the case of transient conditions.
}

The procedure has been implemented into a real-time microcontroller (National Instruments Compact Rio) equipped with a field-programmable gate array (FPGA) bus. The microcontroller is characterized by a $400 \mathrm{MHz}$ real-time processor with 2 GB nonvolatile storage, 128 MB DRAM memory linked with a 3 Mgate FPGA working at $40 \mathrm{MHz}$. The sampling of the voltage waveforms is realized by means of three parallel 16-bits A/D converters that synchronously operate at the FPGA level together with the availability of the UTC-GPS time frame provided by an IRIG-B GPS unit characterized by a time uncertainty of $100 \mathrm{~ns}$.

The synchrophasor estimation procedure involves the following three steps:

1) sampling of the three phase voltages, within a time window $T$ of $80 \mathrm{~ms}$ (i.e., 4 cycles at $50 \mathrm{~Hz}$ ), starting from the UTC-GPS pulse-per-second (PPS) wavefront (1 or 10 PPS) and extraction of the UTC-GPS "nanosecond" value in correspondence of the first voltage waveform sample;

2) reconstruction of the fundamental frequency tone (i.e., a sinusoidal signal characterized by a single frequency) within a specific frequency window $\Delta f$ (i.e., $f_{0} \pm \Delta f$, where $f_{0}$ indicates the rated value of the network frequency);

3 ) estimation of the synchrophasor amplitude, phase and frequency making reference to the reconstructed fundamental frequency tone waveform.

The sampling process (step 1) is performed at the FPGA level of the microcontroller. Therefore, the sampling frequency $f_{\text {samp }}$ of the signal is defined by the operating frequency of the A/D converters at the FPGA level $f_{\mathrm{FPGA}}$ (for the adopted hardware $\left.f_{\mathrm{samp}}=f_{\mathrm{FPGA}} / 2\right)$. As typical values of $f_{\mathrm{FPGA}}$ are in the range of $40-160 \mathrm{kHz}(160 \mathrm{kHz}$ represents the upper limit for the adopted hardware), the sampling frequencies are in the order of $40 \mathrm{kHz}$ to $80 \mathrm{kHz}$. Since the harmonic distortion in distribution network does not reach such very high frequency values, the anti-aliasing filters, applied to the input voltage signals in typical PMU designs, are avoided.

The achieved high sampling frequency value also allows the novel adoption of a reconstruction algorithm of the fundamental frequency tone as the second step of the proposed synchrophasor estimation procedure. In what follows, a description of the implemented fundamental frequency tone reconstruction algorithm is provided.

Input signal $s(t)$ is sampled each $\Delta t=T / N=1 / f_{\text {samp }}$ into a time window $T$ sufficiently short (in our case $80 \mathrm{~ms}$ ) so that the signal can be assumed stationary within $T^{2}$. Signal $s(t)$ can be expressed, in general, with a discrete spectrum as follows:

$$
s(t)=\tilde{s}+\sum_{h=1}^{n} s_{h} \cos \left(h \omega_{0} t+\varphi_{h}\right)+\varepsilon_{t}
$$

where $\tilde{s}$ is the dc component (mean value) of $s(t)$ within the sampled time window $T, s_{h}$ and $\varphi_{h}$ are the amplitude and phase of the $h$ th harmonic component, and $\varepsilon_{t}$ is the Gaussian noise. ${ }^{3}$.

\footnotetext{
${ }^{2}$ Reasonable hypothesis, as we are interested in the analysis of slow transients.

${ }^{3}$ In view of the elevated value of $f_{\mathrm{samp}}$, the maximum number $n$ of harmonic components that could be identified with the adopted sampling frequency largely exceeds the typical harmonic content of the voltage waveforms in distribution systems.
} 
As advised by Grandke in [20], a numerical interpolation technique is applied to improve the accuracy of parameters obtained by the DFT of a generic signal. Also the problem of harmonic interference is essentially removed by the use of such an interpolation. As described next, such a technique provides a first estimates of the following phasor parameters: frequency $f_{0}=\omega_{0} / 2 \pi$, amplitude $s_{1}$, and phase $\varphi_{1}$ of the fundamental frequency tone.

The DFT of (1) can be expressed as follows:

$$
G(k \Delta f)=\sum_{h=1}^{n} S_{h} D_{N}\left[\left(k \Delta f-f_{h}\right) T\right]
$$

where $N$ is the number of samples, $\Delta f=1 / T, \Delta t=$ $T / N, k=0, \ldots(N / 2)-1, S_{h}$ and $f_{\mathrm{h}}$ are the amplitude and frequency of tone $h$, while $D_{N}$ is the so-called Dirichlet kernel defined by

$$
D_{N}(\vartheta)=\frac{\sin (\pi \vartheta)}{N \sin (\pi \vartheta / N)} e^{-\pi i \vartheta \frac{(N-1)}{N}}
$$

being $i$ the imaginary unit.

As already mentioned, the adopted sampling frequency $f_{\text {samp }}$ is in the range between $40 \mathrm{kHz}$ to $80 \mathrm{kHz}$. Therefore, for the considered PMU application, the Nyquist frequency (i.e., $f_{\text {samp }} / 2$ ) can be reasonably assumed to be much larger than the maximum frequency component of $s(t)$ (i.e., $f_{n}$ ) so that the aliasing effects on the signal can be disregarded.

For the identification of the fundamental frequency tone of $s(t)$, two are the problems that need to be addressed, namely:

a) the spectral leakage effects caused by the finite length of time window $T$; and

b) the identification of the correct frequency value that may fall between two subsequent frequency values provided by the DFT.

The algorithm successfully copes with problem a) by applying the convolution the sampled data with the Hanning window

$$
G_{H}(k \Delta f)=\sum_{h=1}^{n} S_{h} H_{N}\left[\left(k \Delta f-f_{h}\right) T\right]
$$

where $H_{N}$ is the Fourier transform of the Hanning windowed data. ${ }^{4}$

Concerning problem $b$ ), the estimate of fundamental tone frequency $f_{0}$ can be expressed as a function of the discrete DFT frequency discretization step $\Delta f$

$$
f_{0}=(m+\Delta b i n) \Delta f
$$

where $\Delta$ bin is the deviation from the true frequency of the estimated fundamental one $(0 \leq \Delta$ bin $<1)$. In (5), $m$ and $m+1$ indicate the two DFT tones that delimit the interval in which $f_{0}$ is assumed to be.

Since the number of samples $N$ per time window $T$ is very large - a condition satisfied by the adopted sampling frequencies, which are much larger than the fundamental tone frequency

$$
\left.{ }^{4} H_{N}(\vartheta)=(1 / 2)\left[D_{N}(\vartheta)-D_{N}(\vartheta+1)+D_{N}(\vartheta-1)\right) /(2)\right] .
$$

(i.e., $50-60 \mathrm{~Hz}$ ) - it is reasonable to approximate the sine function in the denominator of (3) by its argument and assume the following simplification:

$$
e^{-\pi i \frac{N-1}{N}} \approx-1+\frac{\pi i}{N}
$$

As shown in [20], these assumption led to a linear expression of $G_{H}(k \Delta f)$ as a function of $\Delta$ bin. Therefore, $\Delta$ bin can be expressed as

$$
\Delta b i n= \pm \frac{a-2 b}{a+b}
$$

where $a$ and $b$ are the highest and the second highest tone magnitudes in the discrete spectrum of $G_{H}$.

The complex amplitude of the fundamental frequency tone $S_{1}$ at $f_{0}$ is estimated as

$$
\begin{aligned}
& S_{1}=\frac{2 \pi \Delta \operatorname{bin}(1-\Delta b i n)}{\sin (\pi \Delta b i n)} \\
& \times e^{-\pi i \Delta b i n}(1+\Delta b i n) G_{H}(m \Delta f) .
\end{aligned}
$$

The knowledge of $S_{1}$ provides the value of the fundamental frequency tone amplitude $s_{1}$ together with its phase $\varphi_{1}$.

The proposed PMU algorithm reconstructs the time domain fundamental frequency tone as

$$
s_{1}(t)=S_{1} \cos \left(2 \pi f_{0} t+\varphi_{1}\right) .
$$

After such a reconstruction, the value of the phase $\varphi_{1}$ is further estimated as follows:

$$
\varphi_{1}=3 / 2 \pi-2 \pi f_{0}\left[\left(t_{\text {zero-cross }}-t_{1 \text { sample }}\right)+\left(t_{1 \text { sample }}-t_{\text {PPS }}\right)\right] .
$$

In particular, the term $\left(t_{\text {zero-cross }}-t_{1 \text { sample }}\right)$ is the time interval between the first sample $\left(t_{1 \text { sample }}\right)$ of the reconstructed fundamental frequency tone and its subsequent first zero crossing $\left(t_{\text {zero-cross }}\right)$. The zero crossing time is estimated by a linear interpolation between the two consecutive samples before and after the waveform sign change. The reconstruction of the fundamental frequency tone in time domain avoids the presence of multiple zero-crossings so that the term $\left(t_{\text {zero-cross }}-t_{1 \text { sample }}\right)$ is uniquely defined.

A further correction to $\varphi_{1}$ is provided by $\left(t_{1 \text { sample }}-t_{\mathrm{PPS}}\right)$, time interval between the first sample of the reconstructed fundamental frequency tone ( $\left.t_{1 \text { sample }}\right)$ and the time corresponding to the PPS $\left(t_{\mathrm{PPS}}\right)$. This correction allows the achievement of both a substantial independency of the PMU phase uncertainties from $f_{\text {samp }}$ together with a significant reduction of the PMU uncertainty level, as proved by the comparison between the results of the experimental characterization of the developed PMU described below with respect to those, obtained without its implementation.

The experimental characterization of the developed PMU has been carried out by means of reference waveforms generated using a GPS-synchronized function generator, described in [14]. The differences between the reference waveform magnitude and phase with the corresponding values measured by the PMU has 


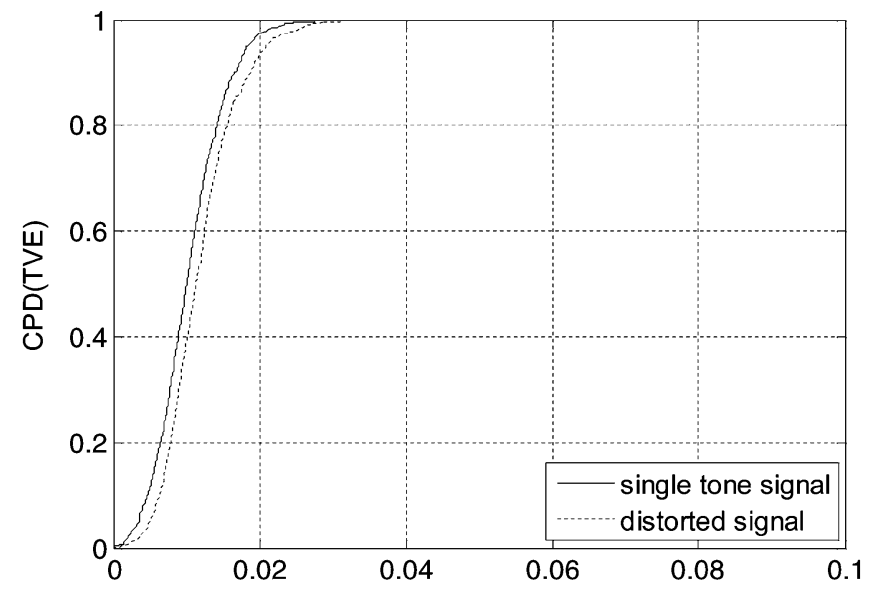

a)

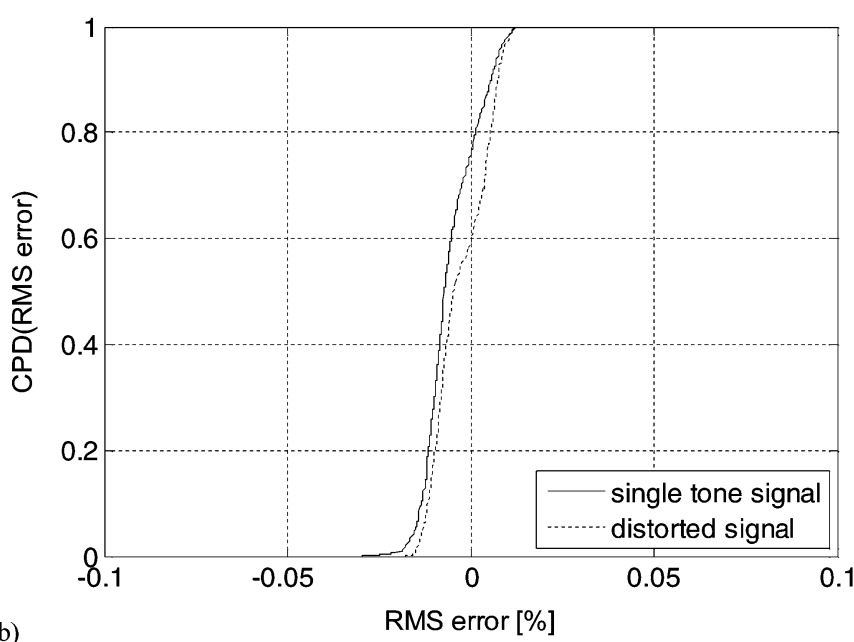

b)

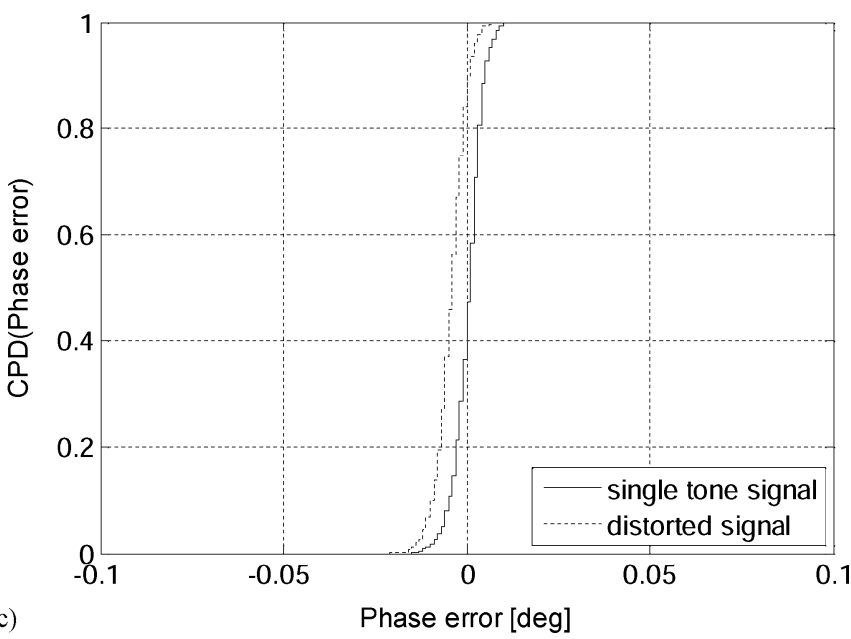

Fig. 1. Cumulative probability distribution functions (CPD) of: (a) TVE, (b) RMS, and (c) phase errors of the synchrophasor estimated by the developed PMU for the case of a single-tone and distorted signals.

allowed us to directly measure the PMU errors, which statistical distributions are reported in Fig. 1. Fig. 1(a) shows the cumulative probability distribution of the TVE, defined in [16], Fig. 1(b) shows the distribution of the synchrophasor estimates RMS error, and Fig. 1(c) shows the distribution of the synchrophasor phase errors. These results refer to both single-tone $(50 \mathrm{~Hz})$ and distorted signals (with spectrum components equal to the limit values provided by the standard EN 50160 [19]).
TABLE I

STANDARD DEVIATIONS OF THE STATISTICAL DistribUtions OF TVE, RMS ERROR, AND SYNCHROPHASOR PHASE ERROR OF FIG. 1

\begin{tabular}{l|c|c|c|c}
\hline \hline \multicolumn{1}{c|}{ Signal type } & TVE & $R M S$ & \multicolumn{2}{|c}{} \\
\hline Single tone & $0.0046 \%$ & $0.0072 \%$ & $3.9 \mathrm{mdeg}$ & $0.069 \mathrm{mrad}$ \\
\hline Distorted signal & $0.0049 \%$ & $0.074 \%$ & $4.0 \mathrm{mdeg}$ & $0.070 \mathrm{mrad}$ \\
\hline \hline
\end{tabular}

The adopted operating conditions of the PMU are the following: $f_{\text {samp }}=80 \mathrm{kHz}$ and $f_{\mathrm{FPGA}}=160 \mathrm{kHz}$.

As shown by Fig. 1, a substantial independence of the PMU performances from the distortion level of the input signal has been obtained.

Table I shows the standard deviations of the TVE, RMS error $\Delta \mathrm{RMS}$, and synchrophasor phase error $\Delta \theta$ distributions of Fig. 1. As defined in [21], these values correspond to the so-called standard uncertainty and prove that the developed PMU are compatible with the requirements of power distribution networks applications.

The computational effort required by the proposed algorithm allows for ten synchrophasor estimates per second with the adopted real time microcontroller.

\section{Description Of THE ACtive Distribution Network}

The experimental tests have been carried out at a $80 \mathrm{MW}$ power plant composed by two aeroderivative gas turbine (GT) units and a steam turbine unit (ST) in combined cycle, connected to a $132 \mathrm{kV}$ substation feeding a urban medium voltage (MV) distribution network.

More in detail, as shown in Fig. 2, the synchronous generators of the three units are connected to a $132 \mathrm{kV}$ power plant substation through $15 / 132 \mathrm{kV}$ step-up transformers. The power plant substation is linked, by means of a cable line, to the $132 \mathrm{kV}$ substation that feeds 15 feeders of the local medium voltage $(15 \mathrm{kV})$ distribution network and provides also the connection with the external transmission network throughout circuit breaker BR1.

Three PMUs have been installed in the subtransmission section of the network (see Fig. 2): one (PMU1) at the power plant substation and two (PMU2 and PMU3) at two sides of circuit breaker BR1. The instrument voltage transformers adopted to connect the PMUs to the power system under study are characterized by uncertainty levels lower than the ones reported in Table I.

The reason for installing the PMUs at the $132 \mathrm{kV}$ section of the system is that such locations allow monitoring the most significant information about the network status, namely, the connection/disconnection status between the system of interest and the external network, and the measurement of the phase angle differences between the phasors at the terminal of $1 \mathrm{~km}$ long cable link connecting the power plant to the distribution substation.

The two GTs are aeroderivative industrial RB211 packages and are equipped with a speed governor, characterized by a 5\% droop and an acceleration limiter and a local frequency integrator (LFI). Each turbine delivers $32 \mathrm{MW}$ mechanical power (at $4850 \mathrm{rpm}$ rated speed and $15^{\circ} \mathrm{C}$ reference temperature) to a $50 \mathrm{~Hz} 32.9 \mathrm{MVA}$ (at $40^{\circ} \mathrm{C}$ cooling) synchronous generator and $93 \mathrm{~kg} / \mathrm{s}$ exhaust mass flow at $509^{\circ} \mathrm{C}$ to a HRSG that generates $10.5 \mathrm{~kg} / \mathrm{s}$ steam flow at $52 \mathrm{bar}(\mathrm{HP})$ and $487^{\circ} \mathrm{C}$, together with $2.5 \mathrm{~kg} / \mathrm{s}$ at $6.5 \mathrm{bar}(\mathrm{LP})$ and $231^{\circ} \mathrm{C}$. The main control valve of 


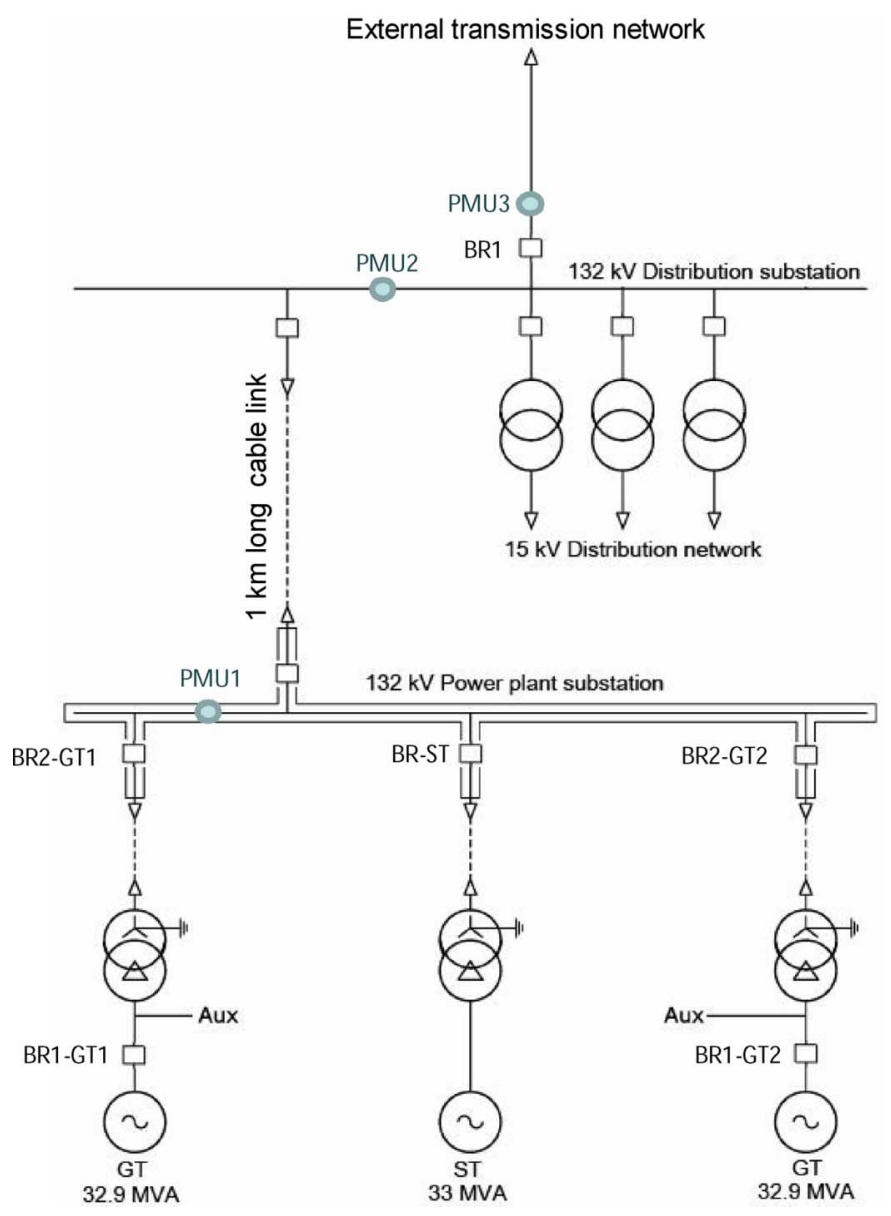

Fig. 2. Scheme of the considered system. PMU1, PMU2, and PMU3 indicate the position of the three installed PMUs.

the 22 MW ST can be regulated in three different operation mode, namely: i) no-load speed control; ii) $p_{\mathrm{HP}}$ pressure control to a constant value; and iii) power and speed regulations. The no-load speed control mode is used during startup and synchronizing maneuvers, while, in normal operating conditions, pressure or power-speed regulation is adopted.

The link between each GT and the ST to its generator is realized by means of a gearbox and each generator is equipped with a brushless exciter.

The power plant is equipped with a power management system (PMS) that: a) operates circuit breaker BR1 for the disconnection of the network from the external grid; b) communicates the so-called Load Droop Anticipator command to the ST control system in case of islanding maneuvers accomplished at rather large power exported levels to the transmission network; c) operates a disconnection of feeders following a predefined priority list in order to guarantee the load balance; d) selects the operation control mode of the two gas turbines (master and slave) for the frequency regulation of the network in islanded conditions; and e) controls the power plant units in order to allow a reliable reconnection maneuvers of the network to the external grid.

Before carrying out the tests that will be described in the following sections, the islanding capabilities of the system of Fig. 2 have been previously analyzed by means of a computer dynamic simulator suitably developed in the EMTP-RV environment which represents both the power plant and the local network with the relevant loads. The main characteristics of the im- plemented models and the analysis of different islanding strategies have been presented in [22].

\section{DESCRIPTION OF THE MANEUVER AND EXPERIMENTAL RESULTS}

Tests have been carried out in two different days, namely on Thursday August 13, 2009 and Friday August 14, 2009 starting at 5 A.M.

\section{A. Test on August 13}

On August 13, at 5 A.M. only GT1 and ST were in operation, at 21.9 MW and 8.1 MW respectively so to almost balance with the load request of the distribution network that was around 29 MW. At around 5:10 the intentional opening of circuit breaker BR1 was operated. The PMS orders the disconnection of two distribution network feeders for a total load of $2 \mathrm{MW}$.

For the case of the above mentioned islanding maneuver, Fig. 3 shows the synchrophasor angle measurements provided by the three installed PMUs for the voltage of each of the three phases L1, L2, and L3.

In order to provide a more concise presentation of the results, in what follows we shall make reference to the positive sequence components of bus voltages only. We have verified that negative-sequence and zero-sequence components are negligible. Fig. 4(a) shows both the angle deviation between the positive sequence components measured by PMU1 and by PMU2, and the analogous angle deviation between the positive sequence components measured by PMU2 and by PMU3. Fig. 4(b) shows the trend of the magnitudes of the positive-sequence components measured by PMU1, PMU2, and PMU3, and Fig. 4(c) the corresponding frequency transients.

As shown in Fig. 4(a), the phase angle deviation between PMU1 and PMU2 does not exhibit a significant change meaning that the active power transit along the cable link between the power plant substation and the distribution substation remained almost constant. Indeed this maneuver has been realized with a negligible power absorption from the external transmission network so that the active power produced by the power plant before the islanding remains basically unvaried after the maneuver. This result is also confirmed by the reduced frequency transient shown in Fig. 4(c). ${ }^{5}$ Quite the reverse, the increasing phase deviation transient between PMU2 and PMU3 of Fig. 4 clearly shows the separation of the islanded grid from the external network.

At the beginning of operation in islanding condition, a fault occurred at a circuit breaker of the distribution substation produced the disconnection of part of the feeders and, consequently, the ST unit shut off. During the following $20 \mathrm{~min}$, unit GT1 fed the islanded network at the frequency of around $50.08 \mathrm{~Hz}$ and at a power level between 20.2 to $20.7 \mathrm{MW}$.

At around 5:35 A.M., a feeder characterized by a too large load was connected, causing the underfrequency trip of GT1 unit that had reached its maximum power output. The data collected by PMU1 and PMU2 are shown in Fig. 5:6 namely: a) the angle deviation between the positive-sequence components of

\footnotetext{
${ }^{5}$ In Fig. 4(c), the frequency transients measured by PMU1 and PMU2 are identical as the corresponding buses are only separated by the cable link.

${ }^{6}$ Data provided by PMU3 are not reported in Fig. 5 as the figure refers to a transient that affected the distribution network when it was operating in islanding conditions.
} 


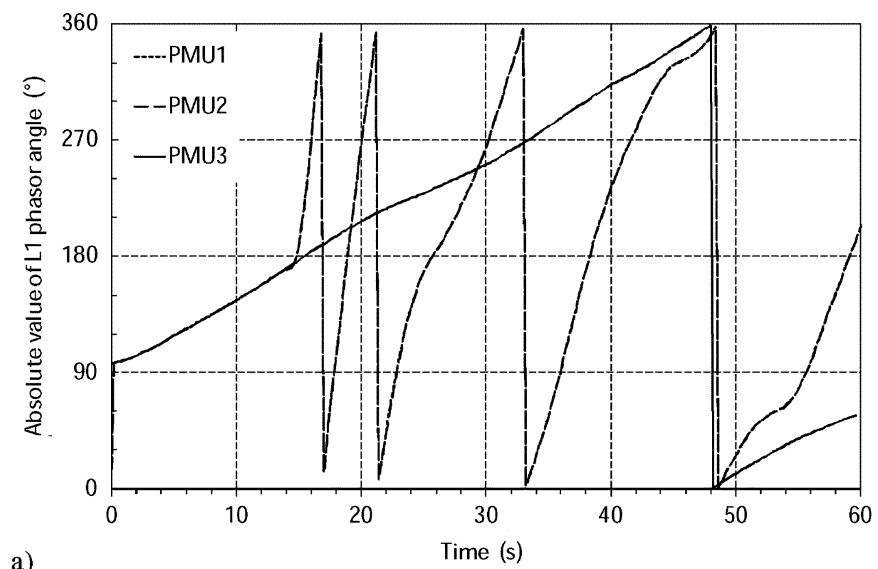

a)

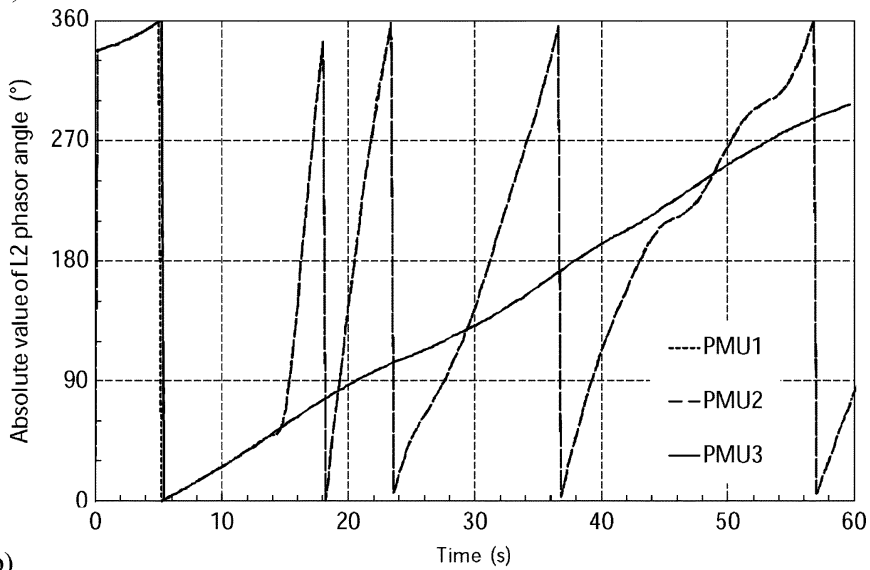

b)

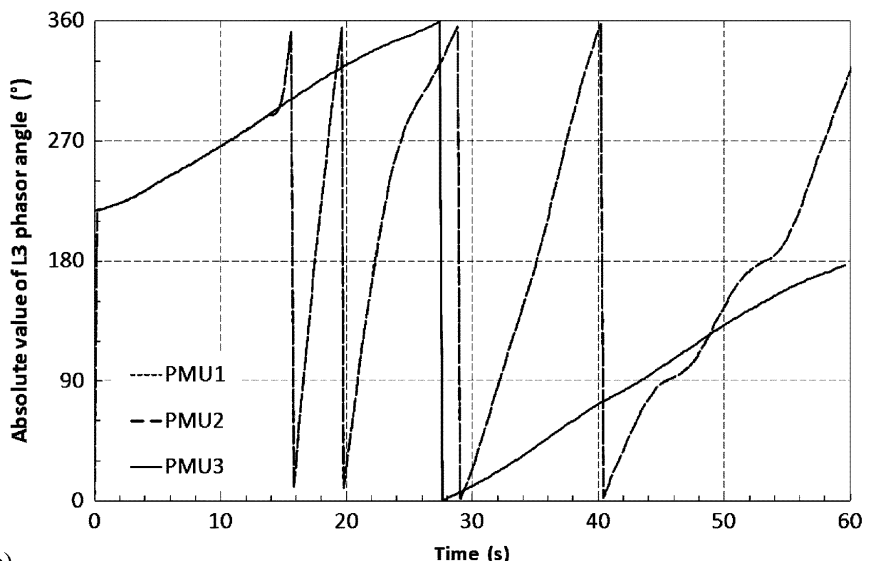

c)

Fig. 3. Estimated absolute values of the phasor angle of: (a) phase L1, (b) phase L2, and (c) phase L3, obtained by the three installed PMUs shown in Fig. 2 during the islanding maneuver on August 13 at 5:10 A.M.

PMU1 and PMU2 phasors; b) the trend of the positive-sequence component magnitudes; and c) the corresponding frequency transients.

\section{B. Test on August 14}

On August 14 at 5:00 A.M., only GT1 unit was in operation at an output level equal to $29.4 \mathrm{MW}$, with a positive export to the external network equal to $1.9 \mathrm{MW}$; GT2 unit was in standby.

At 5:03, circuit breaker BR1 was opened. The data collected by the PMUs are shown in Fig. 6: namely, a) the angle deviations between the positive-sequence components of PMU1 and PMU2 phasors and between those of PMU2 and PMU3 phasors;
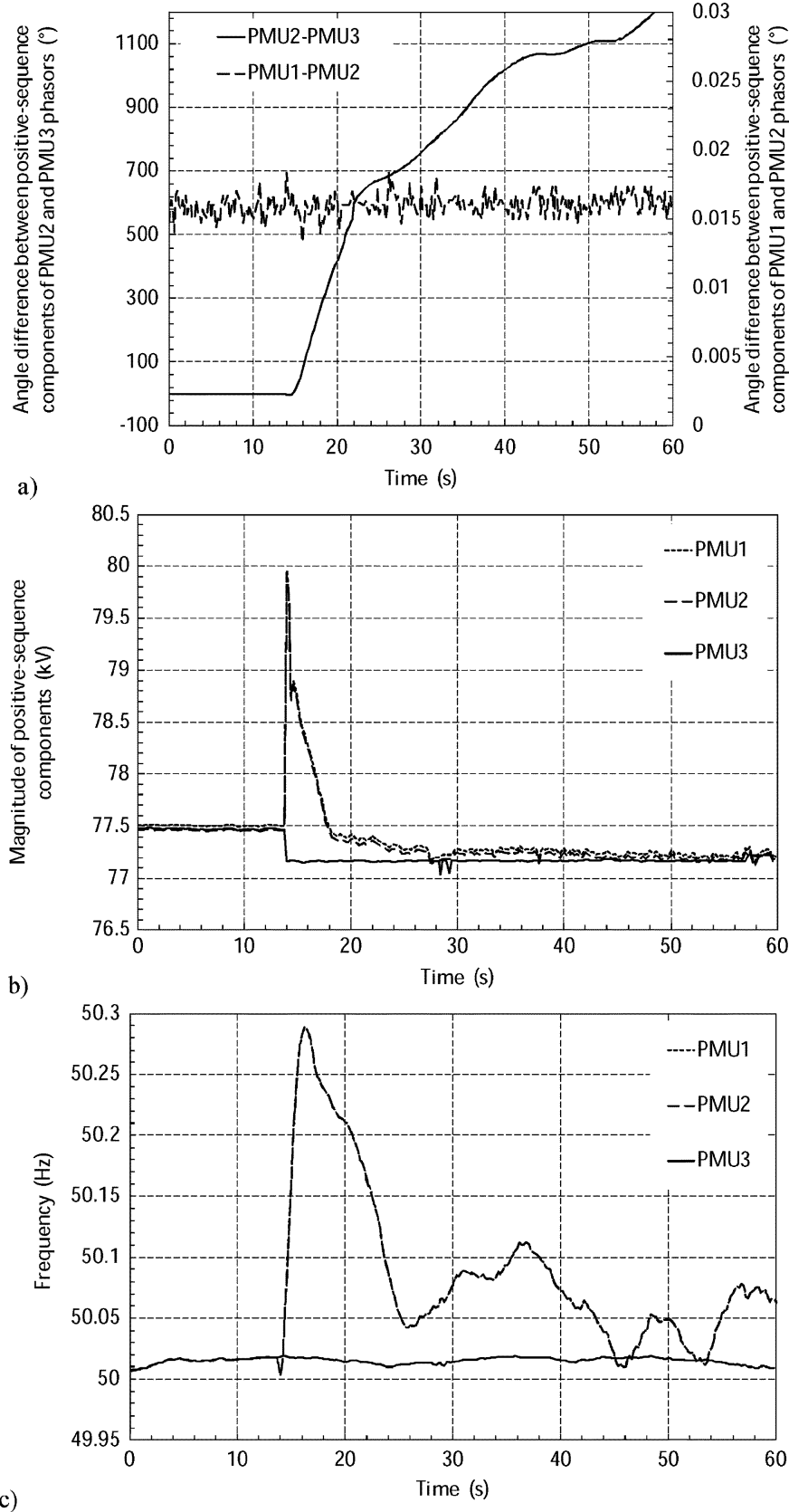

c)

Fig. 4. PMU data collected during the islanding maneuver on August 13 at 5:10 A.M. (a) Angle deviations between positive-sequence components of voltage phasors. (b) Transient of the positive-sequence component magnitudes. (c) Frequency transients.

b) the trend of the positive-sequence magnitudes; and c) the relevant frequency transients measured by the PMUs. Fig. 6(c) also presents the comparison with the generator speed values provided by the SCADA of the power plant. Such a comparison illustrates the accuracy of the developed PMU also during slow transients that involves important frequency deviations from its rated value.

The islanding maneuver in presence of a power export to the external network resulted into a decrease of the power production of the power plant, as shown in Fig. 6(a) by the reduction of the phasors angle deviation between PMU1 and PMU2, i.e., between the beginning and the end of the cable link. Fig. 6(a) also shows an oscillation of the phasors angle deviation between 


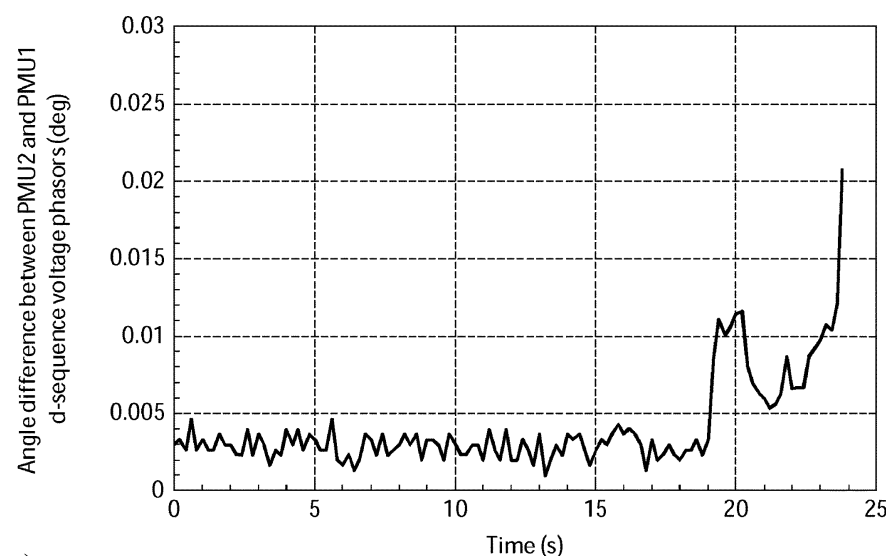

a)

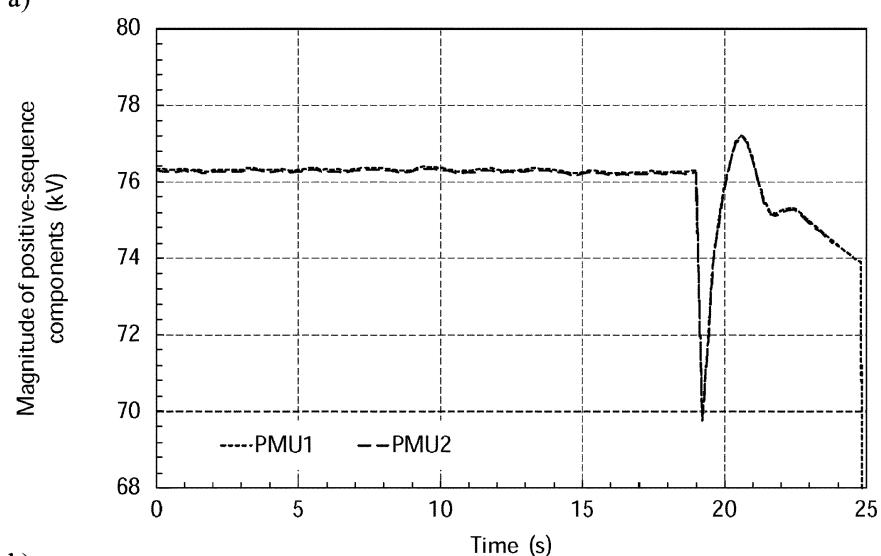

b)

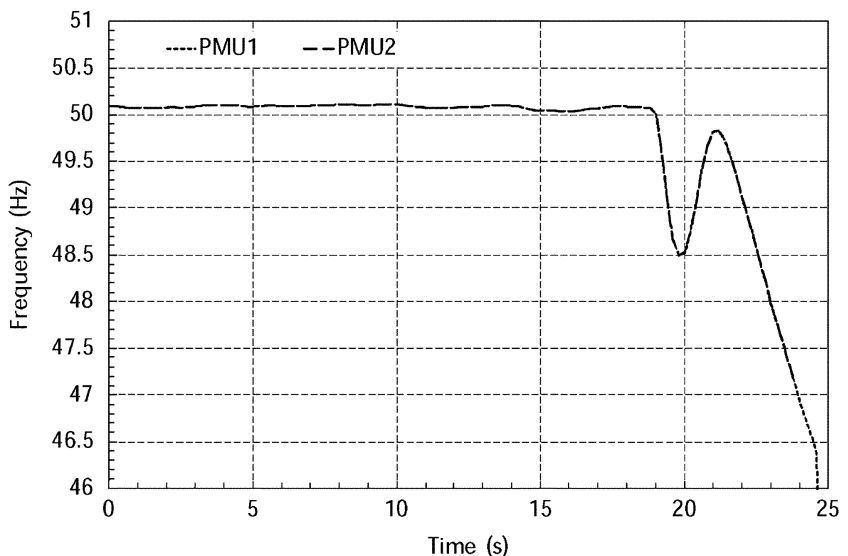

c)

Fig. 5. PMU data collected during the GT1 shutoff for the operation of the underfrequency relay on August 13 at 5:35 A.M. (a) Angle deviation between positive-sequence components of PMU1 and PMU2 voltage phasors. (b) Transient of the positive-sequence component magnitudes. (c) Frequency transients.

PMU1 and PMU2 (in the order of $0.3 \mathrm{~Hz}$ ) associated to the relevant oscillation of the active power exchange between the power plant and the distribution network loads.

The system remained in stable operation. GT1 output was around 27.30 MW and the frequency is maintained at about 50.1 $\mathrm{Hz}$ by the LFI function of incorporated in the GT1 governor.

At around 5:14 the reconnection maneuver was regularly performed. As for the previous cases, Fig. 7 shows the results provided by the installed PMUs during this reconnection maneuver. The synchro-check relay and the synchronizing PMS action permitted the gentle reconnection maneuver illustrated, in particular, by the transient of the angle deviation between PMU2 and PMU3 phasors of Fig. 7(a).
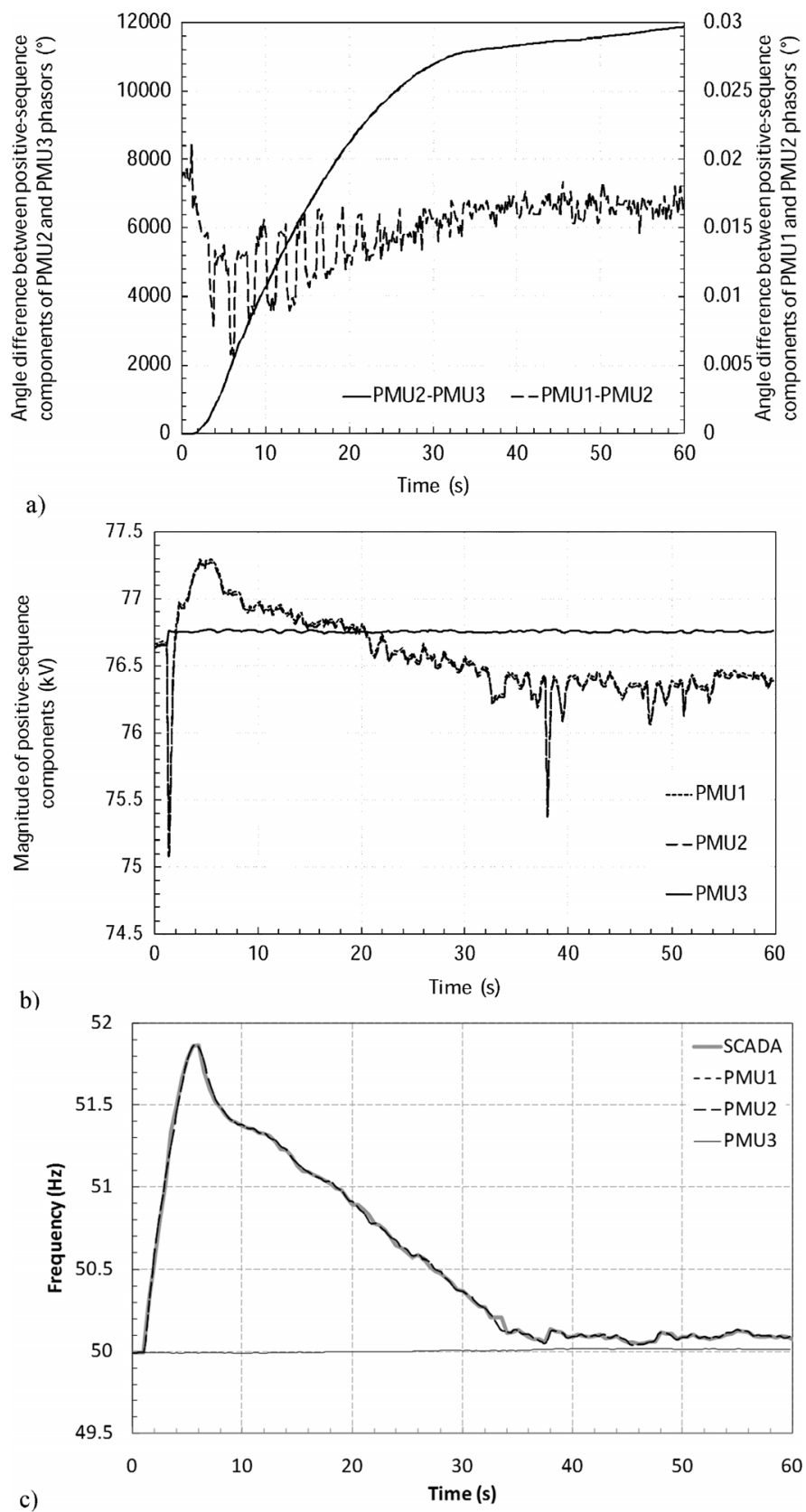

Fig. 6. PMU data collected during the islanding maneuver on August 14 at 5:03 A.M. (a) Angle deviations between positive-sequence components of voltage phasors. (b) Transient of the positive-sequence component magnitudes. (c) Frequency transients.

After the reconnection maneuver to the external grid, unit GT2 was synchronized and brought to an output level of around 27.54 MW at around 6:08, while GT1 output was 29.81 MW and the export to the external grid was $30.42 \mathrm{MW}$.

The power plant PMS was set so to consider GT2 as the master unit; therefore, after a new islanding maneuver at 6:09, GT1 was automatically disconnected by the PMS together with two light-loaded distribution feeders. Unit GT2 remained in stable operation with an output at first of about $28.46 \mathrm{MW}$, decreased to $25.78 \mathrm{MW}$ at $6: 11$.

Fig. 8 shows the results provided by the installed PMUs during the islanding maneuver at 6:09. In this case, at first the frequency of the islanded network decreases with respect to 


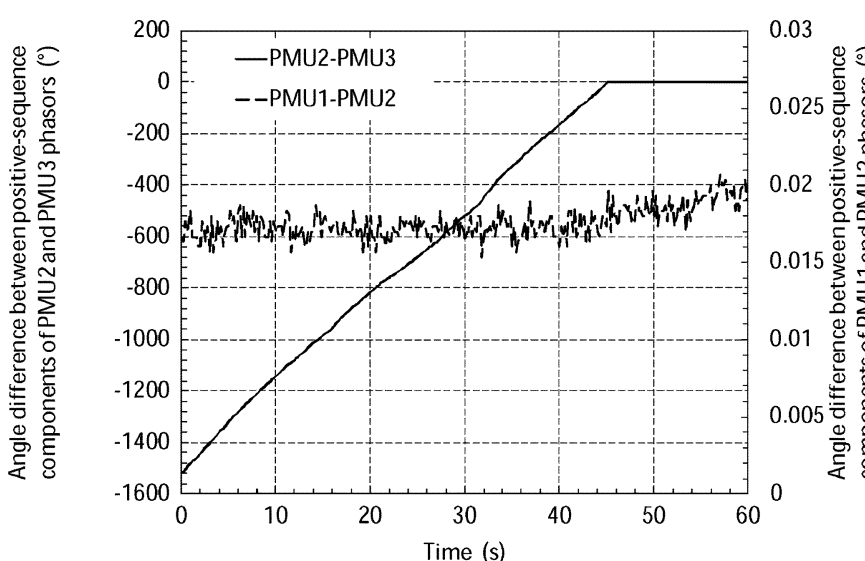

a)

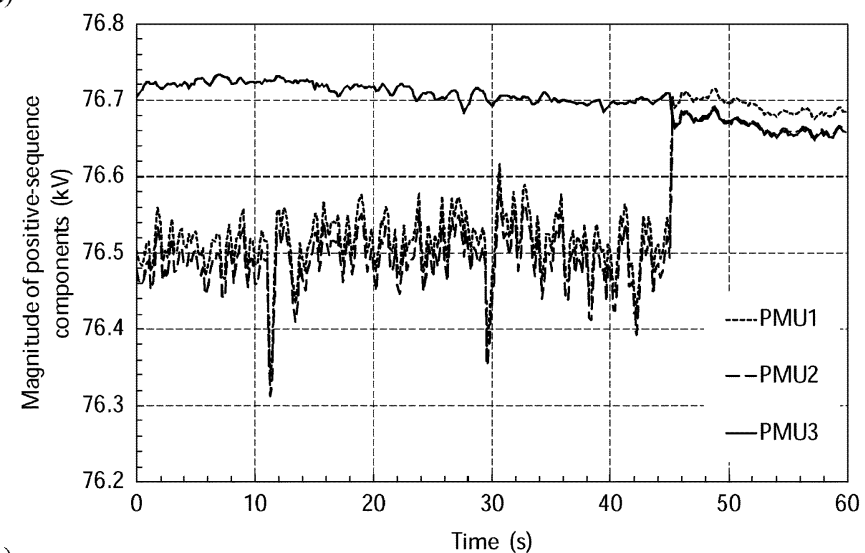

b)

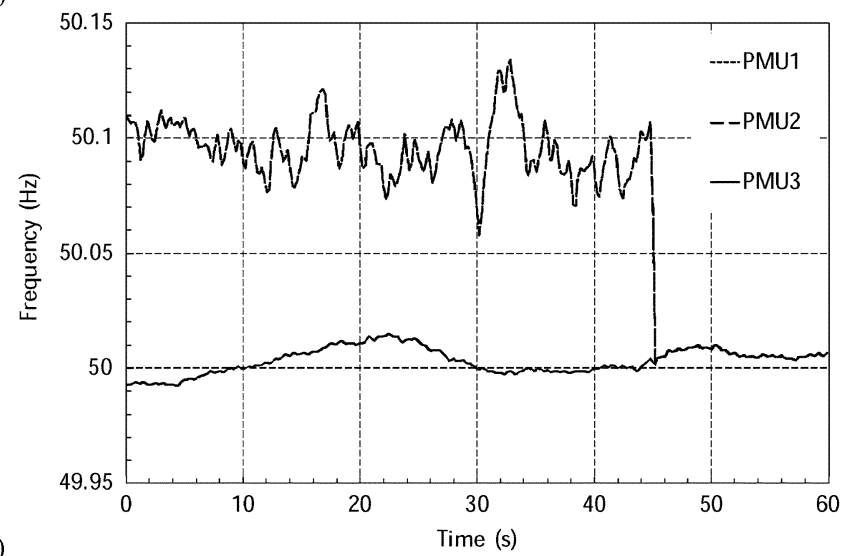

c)

Fig. 7. PMU data collected during the reconnection maneuver on August 14 at 5:14 A.M. (a) Angle deviations between positive-sequence components of voltage phasors. (b) Transient of the positive-sequence component magnitudes. (c) Frequency transients.

the frequency of the external grid, as shown in Fig. 8(c). The GT2 speed governor reacts by increasing the power output as shown in Fig. 8(a) by the increasing, in the first seconds after the maneuver, the angle deviation between PMU1 and PMU2 phasors. Then the LFI action stabilizes the frequency of the islanded network at the predetermined value of about $50.1 \mathrm{~Hz}$. The curve shown in Fig. 8(a), which refers to the phase angle difference between PMU2 and PMU3, is related to frequency transient shown in Fig. 8(c). Indeed, as the frequency difference between the islanded and external networks is negative (Fig. 8(c) between $22 \mathrm{~s}$ and $30 \mathrm{~s}$ ), the relevant phase angle difference is decreasing. As this frequency difference becomes

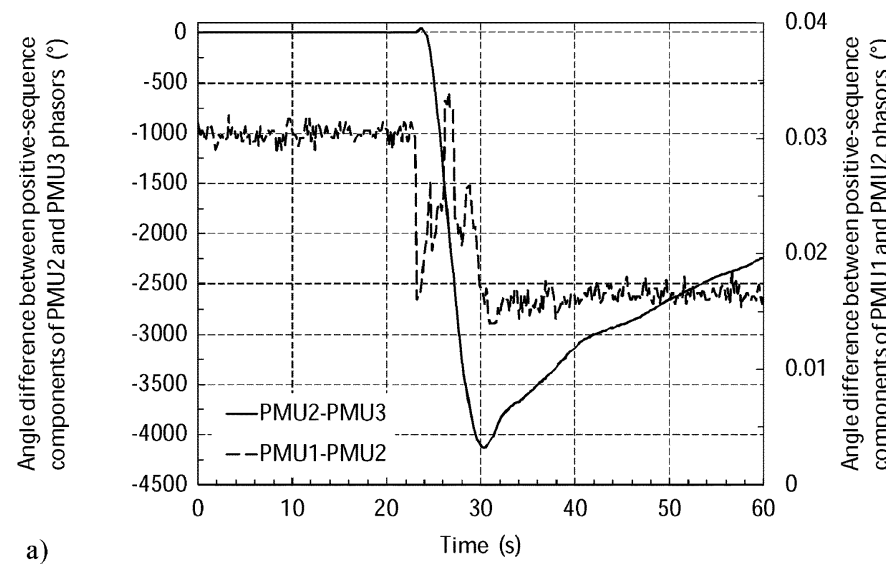

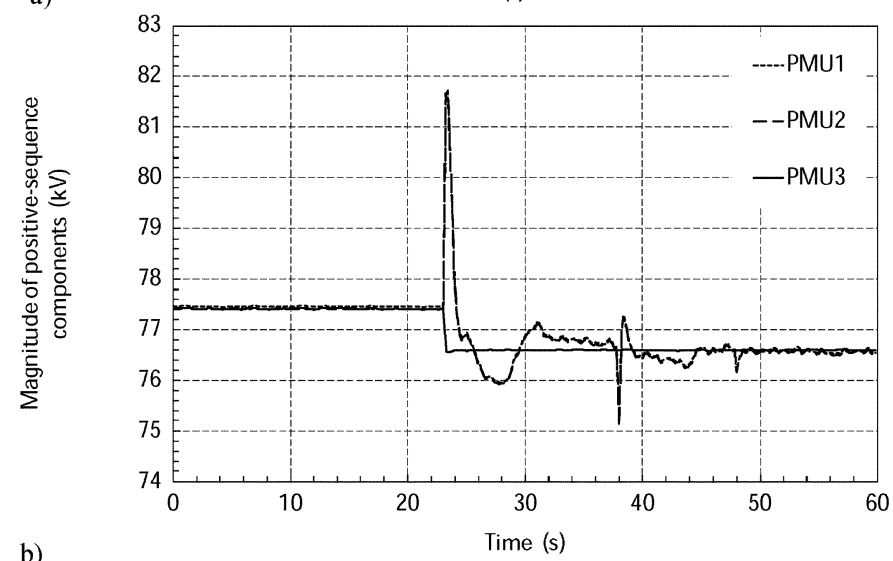

b)

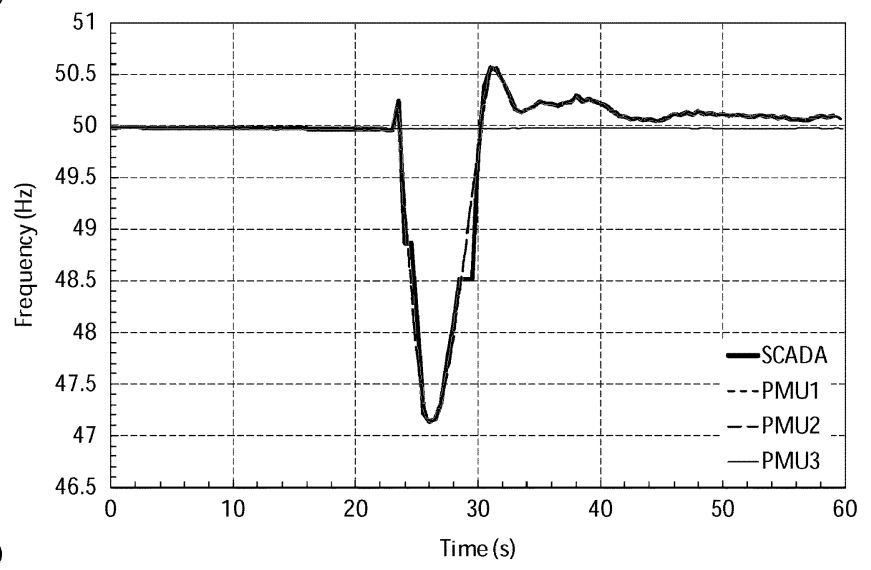

Fig. 8. PMU data collected during the islanding maneuver on August 14 at 6:09 A.M. (a) Angle deviations between positive-sequence components of voltage phasors. (b) Transient of the positive-sequence component magnitudes. (c) Frequency transients.

positive (Fig. 8(c) between $30 \mathrm{~s}$ and $60 \mathrm{~s}$ ) the relevant phase angle difference starts increasing.

At 6:17 the reconnection maneuver of the islanded network to the external grid was performed by the closing of circuit breaker BR1 under the reliable PMS control, as shown also by the PMU results of Fig. 9.

It is worth noting that the islanding maneuver shown in Fig. 8 makes reference to severe operation conditions of the power plant in which, the contemporary disconnection of the GT1 together with two distribution feeders, has resulted into a frequency transient characterized by a minimum frequency of $47.138 \mathrm{~Hz}$ very close to the tripping threshold of the unit minimum frequency relay. 

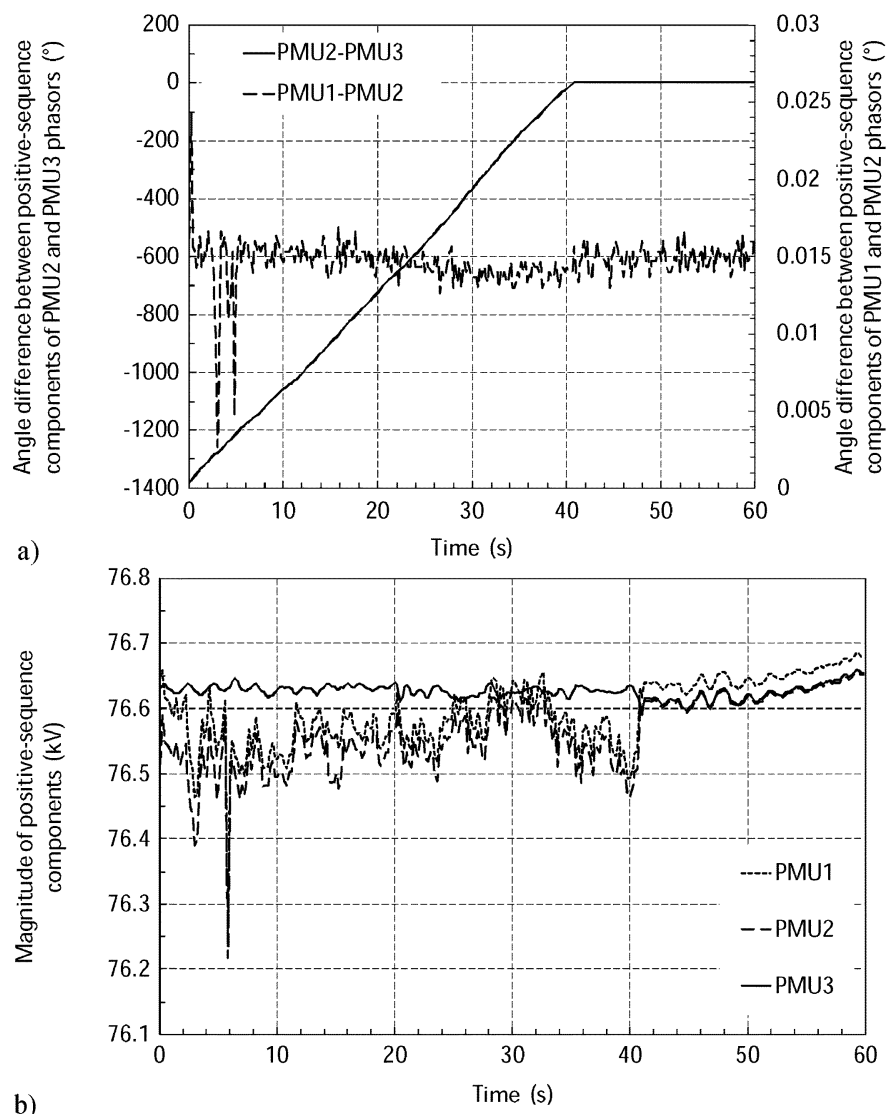

b)

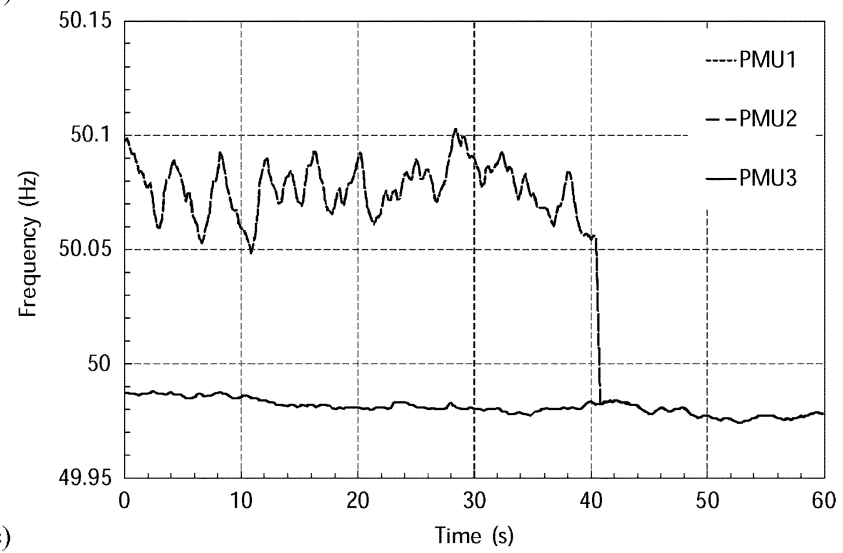

Fig. 9. PMU data collected during the reconnection maneuver on August 14 at 6:17 A.M. (a) Angle deviations between positive-sequence components of voltage phasors. (b) Transient of the positive-sequence component magnitudes. (c) Frequency transients.

Finally, the results of Figs. 6(c) and 8(c) shows that the frequency transients measured by the developed PMU are in good agreement with those recorded by the power plant SCADA demonstrating the ability, as pointed out in Section II, of the developed PMU to be properly used to monitor transients characterized by nonnegligible deviations from the nominal frequency.

\section{CONCLUSION}

As proved by the laboratory experimental characterization of the developed PMUs, based on a DFT tone-reconstruction algorithm, the obtained synchrophasor estimates are characterized by very low values of TVE and of both RMS and phase errors, as required by their application in distribution networks. Moreover, a substantial independence of the PMU performances from the distortion level of the input signal has been achieved.

The developed PMUs have been successfully used during experimental tests aimed at assessing the capability of an urban distribution network to: a) operate autonomously-fed by a local $80 \mathrm{MW}$ combined cycle power plant-after its islanding; and $b$ ) to reconnect to the external grid.

In this framework, the information provided by the PMUs appears to be of great help for the development of improved control and management systems aimed at making these maneuvers more straightforward and reliable. Also, the information coming from the PMUs may represent a useful support to distribution system operator decisions during critical instances experienced by the system, such as islanding/reconnection operation.

Finally, it is worth mentioning that the possibility to measure phase angle differences between voltage phasors at the terminals of short cable links allows for the development of network protection algorithms and procedures based on the measurement of synchrophasors in correspondence of the buses of the distribution system.

The above feature, in addition to the robustness of the overall PMU performances with respect to the distortion level of the measured quantities — not specifically exploited in the present study - makes the proposed PMU particularly suitable for distribution networks.

\section{ACKNOWLEDGMENT}

The authors gratefully acknowledge the fundamental contribution provided by all the power plant operators and distribution network personnel during the experimental tests described in the paper. The authors also thankfully acknowledge A. Mariani for providing the experimental data of the power plant SCADA. The paper takes into account the comments received during the presentation of a preliminary version at the IEEE Innovative Smart Grid Technologies Conference, Gaithersburg, MD, Jan. 19-21, 2010.

\section{REFERENCES}

[1] N. Jenkins, R. Allan, P. Crossley, D. Kirschen, and G. Stcbac, Embedded Generation. $\quad$ London, U.K.: Inst. Elect. Eng., 2000.

[2] J. A. P. Lopes, N. Hatziargyriou, J. Mutalc, P. Djapic, and N. Jenkins, "Integrating distributed generation into electric power systems: A review of drivers, challenges and opportunities," Elect. Power Syst. Res., vol. 77, pp. 1189-1203, 2007.

[3] R. Krebs, E. Lerch, O. Ruhle, S. Gal, F. Lazar, and D. Paunescu, "Future distribution systems with dispersed generation will require network security measures as transmission systems of today," in Proc. IEEE PES Gen. Meet.-Conversion and Delivery of Electrical Energy in the 21st Century, 2008, pp. 1-5.

[4] A. Ishibashi, M. Imai, K. Omata, S. Sato, T. Takagi, Y. Nakachi, and S. Ogawa, "New type of islanding detection system for distributed generation based on voltage angle difference between utility network and distributed generation site," in Proc. 8th IEE Int. Conf. Develop. Power Syst. Protection, 2004, vol. 2, pp. 542-545.

[5] D. M. Laverty, D. J. Morrow, R. J. Best, and P. A. Crossley, "Differential ROCOF relay for loss-of-mains protection of renewable generation using phasor measurement over internet protocol," in Proc. CIGRE/ IEEE Power Energy Soc. Joint Symp. Integr. Wide-Scale Renewable Resources Power Del. Syst., Calgary, AB, Canada, Jul. 29-31, 2009, pp. 1-7. 
[6] O. Samuelsson, M. Hemmingsson, A. H. Nielsen, K. O. H. Pedersen, and J. Rasmussen, "Monitoring of power system events at transmission and distribution level," IEEE Trans. Power Syst., vol. 21, no. 2, pp. $1007-1008,2006$.

[7] J. Tlusty, A. Kasembe, Z. Muller, J. Svec, T. Sykora, A. Popelka, E. V. Mgaya, and O. Diallo, "The monitoring of power system events on transmission and distribution level by the use of phasor measurements units (PMU)," in Proc. 20th Int. Conf. Exhib. Elect. Distrib. (CIRED), Prague, Czech Republic, Jun. 8-11, 2009.

[8] M. Powalko, K. Rudion, P. Komarnicki, and J. Blumschein, "Observability of the distribution system," in Proc. 20th Int. Conf. Exhib. Elect. Distrib. (CIRED), Prague, Jun. 8-11, 2009.

[9] R. J. Best, D. J. Morrow, D. M. Laverty, and P. A. Crossley, "Synchrophasor broadcast over internet protocol for distributed generator synchronization," IEEE Trans. Power Del., to be published.

[10] A. Carta, N. Locci, and C. Muscas, "GPS-based system for the measurement of synchronized harmonic phasors," IEEE Trans. Instrum. Meas., vol. 58, no. 3, pp. 586-593, Mar. 2009.

[11] A. G. Phadke, J. S. Thorp, and M. G. A. Adamiak, "New measurement technique for tracking voltage phasors, local system frequency, and rate of change of frequency," IEEE Trans. Power App. Syst., vol. PAS-102, no. 5, pp. 1025-1038, May 1983.

[12] A. G. Phadke, "Synchronized phasor measurement in power systems," IEEE Comput. Appl. Power, vol. 6, no. 2, pp. 10-15, Apr. 1993.

[13] A. G. Phadke and J. S. Thorp, Synchronized Phasor Measurements and Their Application. New York: Springer, 2008.

[14] M. Paolone, A. Borghetti, and C. A. Nucci, "Development of an RTU for synchrophasors estimation in active distribution networks," in Proc. 2009 IEEE Bucharest PowerTech, Bucharest, Romania, Jun. 28-Jul. 2 2009, pp. 1-6.

[15] A. Carta, N. Locci, C. Muscas, and S. Sulis, "A flexible GPS-based system for synchronized phasor measurement in electric distribution networks," IEEE Trans. Instrum. Meas., vol. 57, no. 11, pp. 2450-2456, Nov. 2008.

[16] IEEE Standard for Synchrophasors for Power Systems, IEEE Std. C37. 118, 2005.

[17] W. Premerlani, B. Kasztenny, and M. Adamiak, "Development and implementation of a synchrophasor estimator capable of measurements under dynamic conditions," IEEE Trans. Power Del., vol. 23, no. 1, pp. 109-123, Jan. 2008.

[18] A. G. Phadke and B. Kasztenny, "Synchronized phasor and frequency measurement under transient conditions," IEEE Trans. Power Del., vol. 24, pp. 89-95, Jan. 2009.

[19] Voltage Characteristics of Electricity Supplied by Public Distribution Systems, Std. EN 50160, CENELEC, 2004, Bruxelles, Belgium.

[20] T. Grandke, "Interpolation algorithms for discrete Fourier transforms of weighted signals," IEEE Trans. Instrum. Meas., vol. IM-32, no. 2, pp. 350-355, Jun. 1983.

[21] "Evaluation of measurement data-Guide to the expression of uncertainty in measurement," Joint Committee for Guides in Metrology (JCGM) p. 100, 2008

[22] A. Borghetti, M. Bosetti, C. A. Nucci, M. Paolone, G. Ciappi, and A. Solari, "Analysis of black-startup and islanding capabilities of a combined cycle power plant," presented at the 43rd Int. Univ. Power Eng. Conf. (UPEC), Padua, Italy, Sep. 1-4, 2008.

Alberto Borghetti (M'97-SM'03) was born in Cesena, Italy, in 1967. He graduated (with honors) in electrical engineering from the University of Bologna, Italy, in 1992.

Since graduation he has been working with the power system group at the same University, where he was appointed Researcher in 1994 and Associate Professor of Electric Power Systems in 2004. His main research interests concern power system analysis, with particular reference to voltage collapse, power system restoration after blackout, electromagnetic transients, and optimal generation scheduling.
Carlo Alberto Nucci (M'91-SM'02-F'07) was born in Bologna, Italy, in 1956. He graduated with honors in electrical engineering from the University of Bologna in 1982.

He was a Researcher in the Power Electrical Engineering Institute in 1983, and a full Professor at the same University, chair of Power Systems, in 2000. He is author or coauthor of more than 200 scientific papers published on reviewed journals or presented at international conferences. His research interests concern power systems transients and dynamics, with particular reference to lightning impact on power lines, system restoration after blackout, and distributed generation.

Prof. Nucci is a Fellow of the IET. He is a Member of the IEEE Working Group "Lightning Performance of Distribution lines"; in CIGRE he serves as chairman of the Study Committee C4 "System Technical performance." He is the IEEE PES Rep. of Region 8. Since January 2010 he is editor in chief of Electric Power System Research.

Mario Paolone (M'07-SM'10) was born in Campobasso, Italy, in 1973. He graduated with honors in electrical engineering (best graduated faculty of engineering award) and received the Ph.D. degree from the University of Bologna, Italy, in 1998 and 2002, respectively.

$\mathrm{He}$ is currently working within the power systems group of the University of Bologna. He is secretary of the CiIGRE Working Group C4.501, "Numerical Electromagnetic Analysis and its Application to Surge Phenomena" and member of the IEEE WG on Lightning performance of distribution lines and of the joint CIGRE-CIRED Working Group "Protection of MV and LV networks against Lightning." He was co-chairperson of the technical committee of the 9th edition of the International Conference of Power Systems Transients (IPST 2009). His research interests are in the area of smart grids, with particular reference to real-time monitoring and operation, power system protections, power system transients, with particular reference to LEMP-interaction with electrical networks and power systems dynamics.

Gaetano Ciappi was born in Barberino Val d'Elsa, Italy, in 1943. He graduated in 1963 in electrotechnical. First he worked in Gruppo Marelli in R\&D department and then in ENEL, engineer department, to process technical specification for power plant, operating rules, troubleshooting in high voltage network, installation of protection and control system in working plant. Since 2001 he is working in HERA, design department, as senior electrical engineer, where he took part in realization of Combined Cycle Cogeneration Plant and Waste To Energy plant. He is responsible for definition of the main features of machinery and electrical system, functional criteria under network normal operating condition and network disrupted, black start up and island mode operating condition, in local medium and high voltage network.

Aurelio Solari was born in Monte Argentario (GR), Italy, in 1953. He graduated in Mechanical Engineering from the University of Pisa, Italy, in 1978. Since then he joined with Nuovo Pignone in Florence, Italy, in Test Department for turbomachines to be installed on Oil \& Gas and Power Plant.

Since 2001 he is working in HERA, design department, as responsible electrical engineer department, where he lead realization of Combined Cycle Cogeneration Plant and Waste To Energy plant. His research interests are power system transients, with particular reference to mechanical behavior of turbo machines and thermal cycle of the plant and their interaction with electrical network. 